\title{
The EUROBALL gamma
}

\section{ray detector array}

\author{
G. de Angelis ${ }^{1}, A$. Bracco $^{2}$ and D. Curien ${ }^{3}$ \\ ${ }^{1}$ Istituto Nazionale di Fisica Nucleare, Laboratori Nazionali di \\ Legnaro, Legnaro, Italy \\ ${ }^{2}$ Dipartimento di Fisica dell'Universita' and Istituto Nazionale di \\ Fisica Nucleare, Sezione di Milano, Milano, Italy \\ ${ }^{3}$ Institut de Recherches Subatomiques, Strasbourg, France
}

7 HE EUROBALL collaboration is a common effort of several $I$ European countries to provide a forefront experimental facility for nuclear structure research. Using composite germanium $(\mathrm{Ge})$ counters, the EUROBALL detector is the prototype of a new generation of gamma-ray detector arrays that has set new limits to gamma-detector technology and provided a step forward both in basic research and in applications. As an example of application outside the nuclear physics domain we should mention the recent discovery by the NASA probe, Mars Odyssey, of large quantities of hydrogen that most probably is bound in water under the Mars surface. This evidence was obtained through neutron and gamma detection, the latter based on the EUROBALL detector technology (encapsulated $\gamma$-ray detectors)-see Fig.1 [1].

The Euroball array consists of $239 \mathrm{Ge}$ crystals geometrically arranged in order to cover $45 \%$ of the total solid angle. Technical details are reported in ref. [2]. Installed at two of the main nuclear structure facilities in Europe, at LNL (Legnaro-Italy) and at IReS-Vivitron (Strasbourg-France), see fig. 2 , it has allowed the investigation of atomic nuclei at extreme conditions of angular momentum values and of proton/neutron ratios.

This paper will give an overview of the research activities by selecting a number of examples. We, the authors, would like to dedicate this article to the memory of our IReS colleague and good friend Dr. Jean-Pierre Vivien who has untimely and brutally passed away when this paper was under discussion.

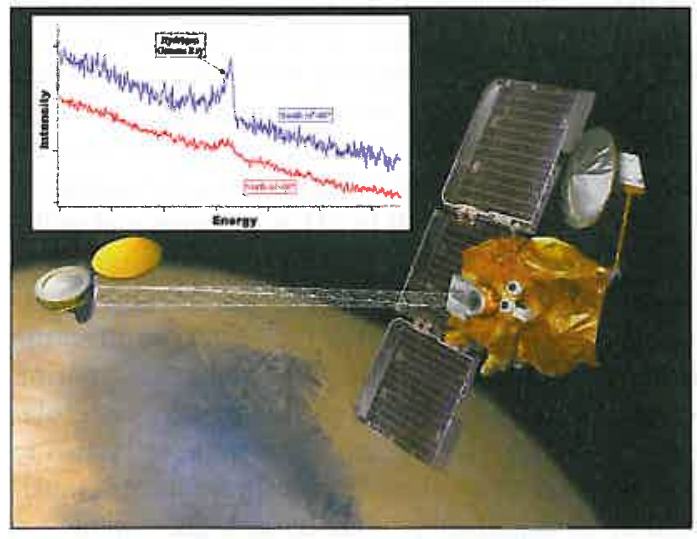

$\Delta$ Fig. 1: Mapping elements through $\gamma$-ray detection using the Mars Odyssey satellite. Neutrons and $\gamma$-rays are produced by the interaction of cosmic rays on Mars. The inset is a portion of the Odyssey $\gamma$ spectra showing the emission line due to the capture of thermal neutrons by hydrogen. The $\gamma$ counter uses the incapsulation technology developed for EUROBALL.

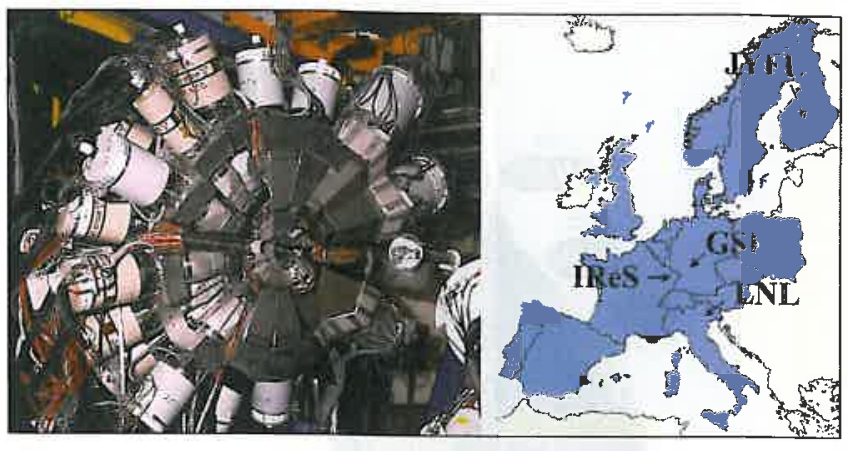

A. Fig. 2: The EUROBALL gamma ray detector array, presently at IReS (F), has been previously operated at LNL (I). Parts of the detector will be reassembled at GSI (D) for being used with beams of radioactive ions (RISING project) and at LNL (I) coupled to a magnetic spectrometer (PRISMA) to exploit the high intensity stable beams provided by the accelerator complex of the laboratory. The remaining detectors will move to JYFL, Jyväskylä (Finland) to complement the Jurogam array.

\section{Why study nuclei at extreme conditions?}

The atomic nucleus is the paradigm of a mesoscopic system (finite many-body system) of strongly interacting Fermions where quantal size effects play a central role. Renormalization effects for the nucleon-nucleon interaction are strongly boosted by making the system more polarizable, that is less bound. A detailed investigation of the nuclear structure of nuclei at extreme conditions, both at the limit of angular momentum and of isotopic spin (neutron/proton ratio) where specific parts of the nuclear forces are strongly amplified, allows definitive tests to be carried out on the effective interaction acting between nucleons in nuclei. These studies provide the basis for a first principle description of the nuclear structure and are necessary to set stringent limits on fundamental symmetry-violation effects and on the lifetimes of rare processes. With an experimental sensitivity up to $10^{-5}$ of the production cross section, such as that of Euroball, it has been possible to provide an unprecedented study of the nuclear properties under extreme conditions by addressing a number of problems concerning high spins and nuclei far from stability.

\section{Nuclear Structure at the limits of the angular momentum}

Through the response of the nucleus to rotational stress one can investigate a wide variety of nuclear structure phenomena showing the different facets of a finite fermionic system. In spite of the fact that rotating nuclei have been studied since the early 50 's, many new phenomena have been encountered and open questions still remain in this field. Particularly intriguing is the existence of very elongated nuclear shapes and the appearance of new degrees of freedom (unusual symmetries like tetrahedral or octahedral) [3].

The stabilization of very exotic shapes at high angular momentum not only provides unique information on the detailed structure of the nuclear potential but also allows one to infer the underlying symmetries characterizing the dynamical system. Breaking of the rotational symmetry can be related to asymmetries in charge distribution (related to deformed shapes as for macroscopic objects) or to current (only quantal) distribution. The most common are prolate elongated axial-symmetric shapes. Prolate axial-symmetric structures with approximately 2:1 and 3:1 axis ratios are called super- (SD) and hyper- (HD) deformed-see figure 3. For these highly deformed structures, 


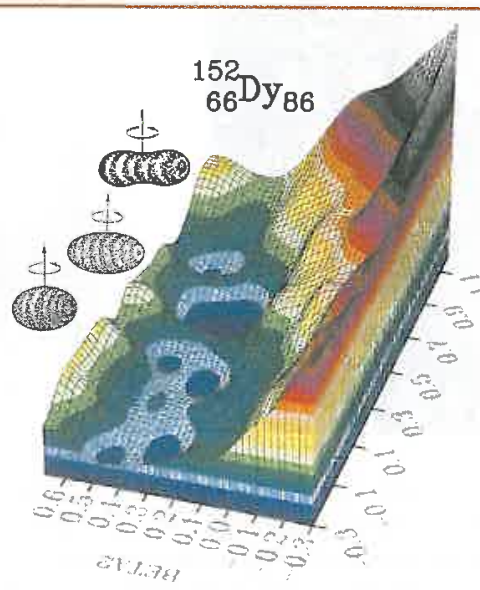

4. Fig. 3: Potential energy surface as a function of the quadrupole deformation, $\left(\beta_{2} \cos (30+\gamma)\right)$ and $\left(\beta_{2} \sin (30+\gamma)\right)$, calculated for the very elongated structures in ${ }^{152} \mathrm{Dy}$. One notices the very pronounced minima corresponding to superdeformed (SD) and hyperdeformed (HD) shapes coexisting with the normal deformed (ND) shape of the nucleus.

information on the properties and the behavior of the nucleus is contained in a cascade of about thirty gamma-rays emitted as it de-excites to its ground state from the highly excited state in which it was produced. With the EUROBALL array, designed to pick out high multiplicity cascades of $\gamma$-rays, discrete nuclear states up to the fission limit have been identified. Beyond the region of discrete states, studies of the high-spin quasi-continuum have brought information on the order-chaos transitions. In particular, in connection with superdeformation, which has become one of the areas at the forefront in nuclear structure, the problems of the feeding and decay-out of the SD bands have attracted particular interest. Concerning the problem of the feeding of superdeformed configurations, it is important to mention that indications on the $\gamma$-decay of the giant dipole resonance built on SD excited states have been recently obtained [4]. Below we describe in more detail results showing the first evidence for non-axially-symmetric superdeformed shapes and the status for the search for hyperdeformation.

\section{Triaxiality and wobbling motion}

Nuclei when rotating may also develop shapes in which the axial symmetry is broken [5]. In contrast to the region around the ground states where deformed nuclei are known to be of axialsymmetric quadrupole type, at high spin, because of the effect of the Coriolis and centrifugal forces, one expects a considerable deviation from the axial-symmetric shapes-see figure 4 . In principle any elongated nucleus can be treated as a quantum rotor that can be brought into rotational motion in a nuclear reaction. In a quantum description only discrete energy values can be associated with the rotational motion, the lowest energy usually corresponding to the most regular rotation. For an even-even elongated nucleus such a rotation corresponds to the direction of angular momentum that is perpendicular to the geometrical elongation axis. Any further intrinsic excitation of the system must lead to a perturbation of such a regularity and in particular some excited individual-nucleons that tend to align their angular momenta with the axis of collective rotation $\mathrm{R}$ will necessarily influence the original regularity of the motion: the regularity will be lost. A classical image of such a situation is known from the

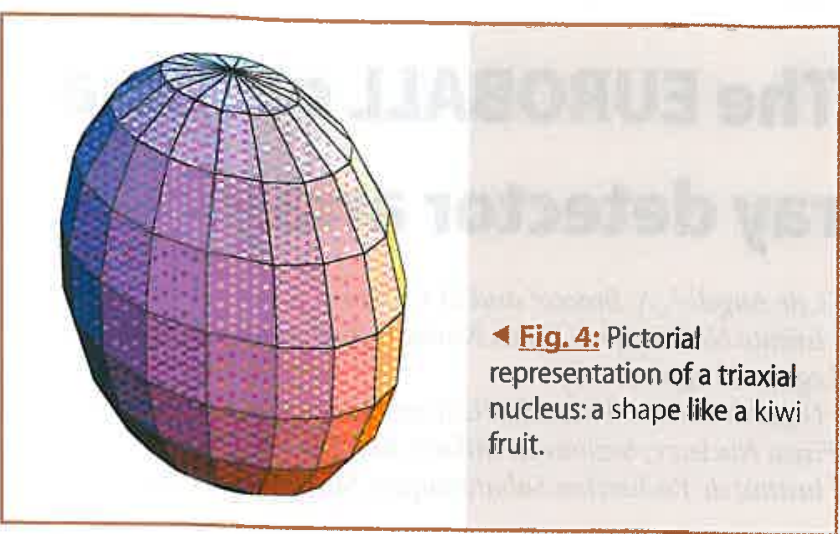

studies of the rigid top that, under specific initial conditions, produces characteristic precession phenomena. Generally one may associate the word "wobbling" with the forms of the motion accompanying such excitations.

It represents a small amplitude fluctuation of the rotational axis away from the principal axis such as a precession motion with the largest moment of inertia, which translates into the "zigzag" pattern of the gamma transition probabilities. This wobbling mode is similar to another collective mode-the $\gamma$ vibrationassociated with the transition from an axially symmetric to a triaxial mean field in the non-rotating case. Excitations of the wobbling mode was predicted long ago and have recently been identified due to the high sensitivity of EUROBALL $[6,7]$.

\section{Search for hyperdeformation}

The nuclear many-body system may, under the influence of rotational induced forces, develop various exotic shapes. Most appealing is the strongly elongated, axially symmetric hyperdeformed (HD) shape with principal axis ratio 3:1, which is predicted in different regions but is still not fully experimentally identified. Much emphasis has been devoted to studies of the interplay between reaction dynamics, binding energies and fission barriers to optimize the population of HD structures at the limits of reachable angular momentum. Nuclei in mass regions where experiments are ongoing are $\mathrm{Ba}, \mathrm{Xe}$ and $\mathrm{Sn}$. Traces of structures in the decay pattern which are presumably generated by nuclei of very elongated shapes were recently observed-see Fig. 5.

In figure 5 an energy matrix of $\gamma-\gamma$ coincidences is sliced on a plane orthogonal to the main diagonal. In such a representation $\gamma$-ray cascades with a definite moment of inertia appear as a pair of energy peaks equally spaced (modulus an integer number). with respect to the main diagonal (ridge structure). The distance between such peaks (shown by the arrows and corresponding to $2 n W$ equal to $105(n=1)$, or to $210(n=2)$, is proportional to the moment of inertia (here $\mathrm{J} \sim 75 \hbar^{2} \mathrm{MeV}^{-1}$ ). Such a value of the moment of inertia corresponds to a 3:1 axis ratio. Ongoing experimental work is expected to clarify better the existence and nature of these structures. In fact, the problem of hyperdeformation remains open also in view of the fact that another possible explanation for the observed weak ridge structure could be related to an oblate-prolate phase shape transition (called a Jacobi transition) predicted by the liquid drop model at the highest spins.

\section{Cluster and molecular structures in nuclei}

Very elongated shapes are also predicted in light nuclei. The most exotic examples involve $\alpha$-particles and ${ }^{12} \mathrm{C}$ or ${ }^{16} \mathrm{O}$ clusters as substructures. The existence of cluster deformed shapes is deduced mainly from the observation, in light symmetric systems, of resonances in binary reaction channels. Such molecular structures 


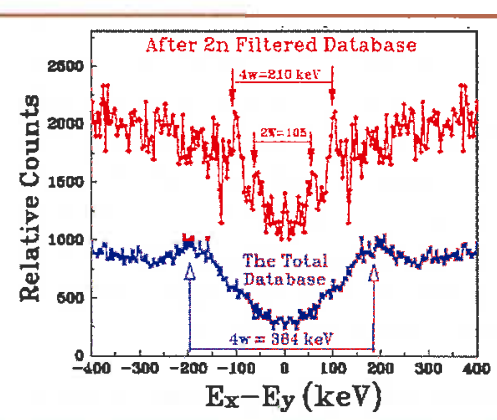

Aig. 5: Gamma spectra of ${ }^{126}$ Ba obtained requiring coincidences of equally spaced gamma-rays ( $E x-E y=k)$. The presence of peaks in the red top spectrum, after filtering on highfold events, is here a signature of rotational structures with constant moment of inertia. The moment of inertia can be deduced by the energy separation of the pairs of peaks. It is compatible with a 3:1 axis ratio and is a possible indication of HD structure at high angular momentum.

also give rise to exotic shapes like reflection asymmetric molecular structures and to strongly deformed isomeric states consisting of clusters and loosely bound neutrons in multi-centre orbits [8].

\section{Breaking the rotational symmetry by current distributions}

Until recently it was thought that near-spherical nuclei always emitted irregular patterns of $\gamma$-rays. However, very regular patterns in the energies of $\gamma$-ray cascades-and hence possible evidence for rotation-were detected in nuclei that were known to be almost perfect spheres [9]. For these cases most of the angular momentum of the nucleus is generated by just a few of the valence protons and neutrons, whose coupling is governed by the overlap of the wave-functions that represent the distribution of nucleon density in the nucleus. Their configuration can be thought of as an unisotropic arrangement of crossed "current" loops embedded in the spherical mass distribution of the nucleus-figure 6. An orientation axis is defined along the total angular momentum vector, $J$, and the system can rotate about this axis. This behavior, investigated also at the EUROBALL spectrometer [10], has been termed "magnetic rotation" because the rotational sequences of states (and transitions) arise from the anisotropy of currents in the nucleus, which produce a magnetic moment [11]. In comparison, the more familiar rotation of deformed nuclei (and molecules) could be named "electric rotation" to reflect the fact that it results from an anisotropy in the charge (and mass) distribution.
A related topic is the spontaneous chiral symmetry breaking (giving rise to left- and right-handed systems as in molecules) which has been discovered in odd-odd nuclei having triaxial shapes. It is based on the fact that three spatial orientations are different, namely configurations where the angular momentum vectors, i) of the valence proton, ii) of the valence neutron and iii) of the core rotation, are mutually perpendicular. Such angular momentum vectors can form a left- and a right-handed system, related by the chiral operator, which combines time reversal and rotation by 180 degree. Spontaneous chiral symmetry breaking in the body-fixed frame is manifested in the laboratory frame as almost degenerate doublets of gamma transitions in $\Delta I=1 \quad(P=1)$ bands-see right hand side of figure 6.

\section{Nuclear Structure at the limits of the isotopic spin}

The most critical quantities in determining the predicted properties of a nucleus from a given effective interaction, is the overall number of nucleons and the ratio $\mathrm{N} / \mathrm{Z}$ of neutrons to protons. It is the extremes in these quantities, which define the limits of existence for nuclear matter. This new field of research in nuclear physics will be opened up by the second generation radioactive beam accelerators. In the case of nuclei not too far away from the valley of stability, there is the possibility to investigate them using high intensity beams of stable ions in combination with very efficient detectors. A large part of the EUROBALL experimental program has been dedicated to the investigation of the structure of proton-rich nuclei through a large variety of dedicated ancillary detectors for reaction products.

\section{Selfconjugate nuclei and mirror symmetries}

Isospin symmetry is a consequence of the approximate charge invariance of the nucleon - nucleon forces. The isospin symmetry explains the nearly identical energy spectra observed in pairs of mirror nuclei. The main contribution to the isospin symmetry breaking is the Coulomb interaction. Although the symmetry is already broken to some extent, at the level of the strong interaction, and to a much larger extent by electromagnetic forces, the isospin formalism remains a very powerful tool.

Isospin symmetry allows one to relate the properties of corresponding levels in different nuclei, from which complementary information can be derived on the structure of the nuclear wave function. The energy differences of analog states along rotational bands in mirror nuclei-mirror energy difference (MED) - have been investigated in the last few years in the $f_{7 / 2}$-shell in the mass region between $A=40-60$ and for heavier nuclei. By resorting to large scale shell model calculations the mechanism of the back-

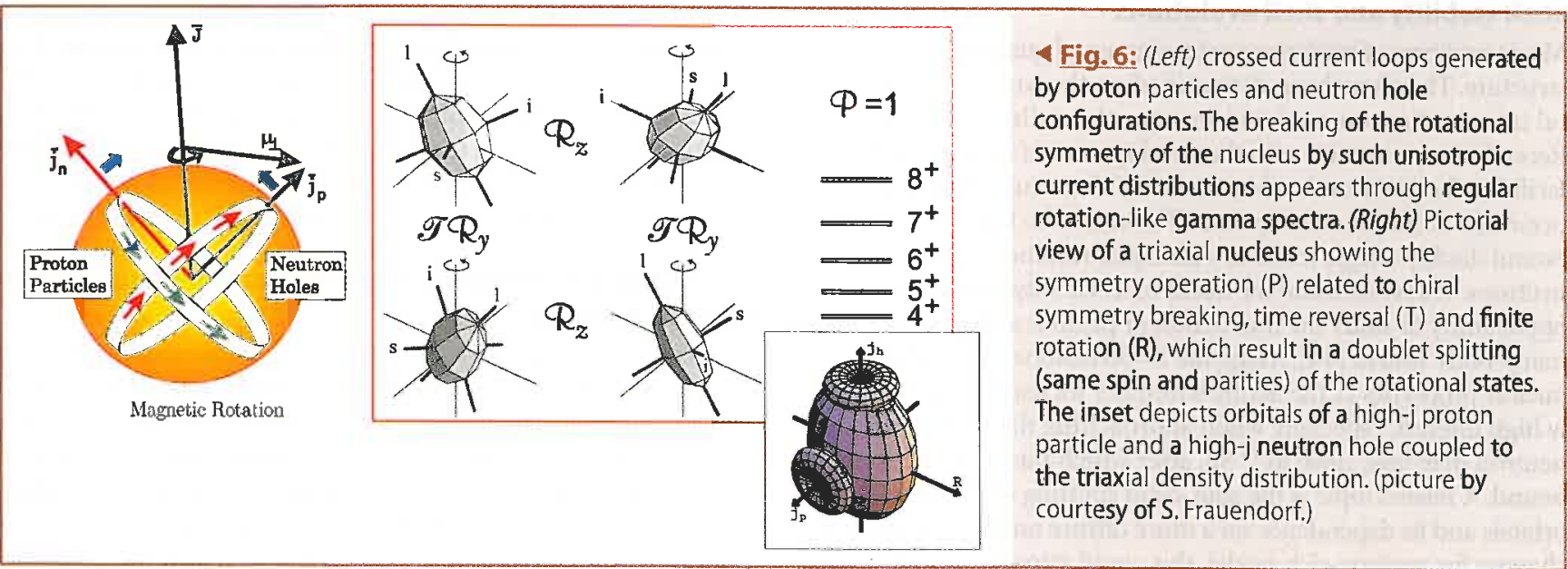




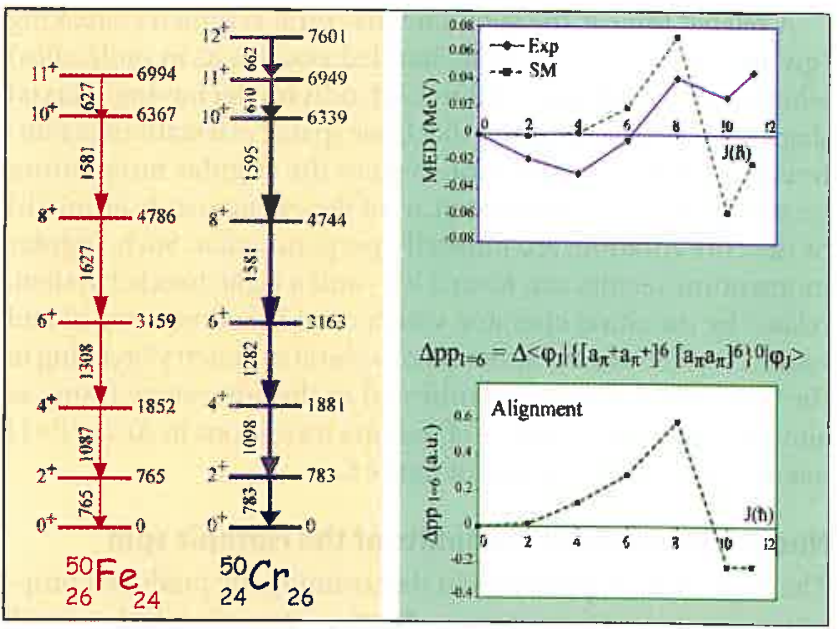

$\triangle$ Fig. 7: (Left) excited states of ${ }^{50} \mathrm{Fe}-{ }^{50} \mathrm{Cr}$ mirror nuclei investigated with EUROBALL. (Right) The upper part of the figure shows the measured (Exp) and calculated (SM) energy differences for mirror nuclei as a function of the angular momentum. The maximum of the curve indicates an alignment process of a pair of nucleons. The lower part of the figure shows the average number of proton pairs in the maximal aligned configuration (by Shell Model calculations).

bending in rotating mirror nuclei has been related to the MED and explained in terms of the alignment of like-nucleon pairssee figure 7. It has also been shown that the MED gives information on the evolution of nuclear radii along the yrast bands and provides direct evidence for charge-symmetry breaking of the nuclear field [12].

\section{Isospin Symmetry}

The consistency of the standard model for electro-weak interaction can be checked through the unitarity test of the Cabibbo-Kobayashi-Maskawa (CMK) matrix. New data, based on super-allowed Fermi $\beta$-decay rates, suggest that the CMK-matrix fails the unitarity test, pointing to physics beyond the Standard Model. These conclusions partly rely on calculations of the corrections for nuclear isospin purity of heavy $\mathrm{N}=\mathrm{Z}$ nuclei. The size of the isospin mixing in low energy nuclear states has recently been determined in the mass $A \approx 60$ region through the precise measurement of the strength of isospin forbidden gamma transitions, namely $\mathrm{E} 1$ decays in $\mathrm{N}=\mathrm{Z}$ nuclei [13].

\section{Shell stability and shell evolutions}

Magic numbers of nucleons are fundamental quantities in nuclear structure. They have been determined on the basis of experimental information on nuclei in or near the valley of $\beta$-stability. Recently, in connection with the development of radioactive beam facilities, the question has been raised if the same magic numbers persist far from the stability line. ${ }^{100}{ }_{50} \mathrm{Sn}_{50}$ is the heaviest particlebound doubly-magic nucleus with equal number of protons and neutrons. This system and the nuclei in its vicinity provide a unique opportunity to study the interaction of protons and neutrons in a many-body system [14]. Along the $\mathrm{Z}=50$ isotopes the evolution of nuclear properties as the neutron number increases is of extremely high interest, especially when approaching the other limit, the neutron drip line, close to ${ }^{150} \mathrm{Sn}$, after which nuclei are no longer bound. A related topic is the spin-orbit splitting of the mean-field orbitals and its dependence on a more diffuse nuclear surface. If it changes for neutron rich nuclei, this could strongly influence the magic numbers for nuclei far from the stability line. Radioactive ion beams will therefore play a very important role in investigating nuclear properties far from stability. Spectroscopic studies of neutron-rich nuclei not very far from the stability line can at the moment also be performed through deep inelastic reactions, where several nucleons are interchanged between projectile and target. Information has been obtained with EUROBALL by using $\gamma$-ray coincidences between the two partners (target- and projectile-like) for the identification of unknown $\gamma$-decays.

\section{Future perspectives with gamma detector arrays}

Present gamma detector arrays are already very sophisticated instruments able to search for very weak reaction channels. New perspectives in the field of nuclear structure will be opened both by the use of high intensity beams of stable ions combined with an increasing selectivity of the instrument, as well as by the availability of moderately intense beams of radioactive ions. The combined use of $\boldsymbol{\gamma}$-ray detector arrays and of magnetic spectrometers for reaction channel selection has been successfully exploited in the past and will be further developed at Jyväskylä in Finland and at LNL where EUROBALL detectors will be used to study the level structure of exotic nuclei identified respectively by the RITU gas filled separator and by the PRISMA magnetic spectrometer.

\section{Nuclear Structure perspectives using high intensity beams of stable ions}

High intensity beams of stable ions offer an interesting possibility to extend our knowledge of nuclear structure both for proton-rich and moderately neutron-rich nuclei. Quasi-elastic or multi-nucleon transfer reactions can be used to populate moderate neutron-rich nuclei along shell closures where nuclear structure calculations predict radical changes in the shell structure. Particularly powerful is the combination of large-acceptance high-resolution magnetic spectrometers with highly segmented Ge detector arrays. An high granularity of the $\gamma$-ray detector array provides for a good $\gamma$-energy resolution allowing a proper treatment of the Doppler effect. A system combining the new magnetic spectrometer (PRISMA) with the composite Ge detectors (Clovers) of EUROBALL is now coming into operation at LNL in Italy using the high intensity stable ion beams of the accelerator complex (PIAVE-ALPI).

\section{Radioactive ion beams and gamma spectroscopy}

The use of beams of unstable ions will finally allow nuclear structure studies for the most exotic systems. We should mention here the future EUROBALL detector campaign at GSI (Germany), the Rising project. Gamma-ray spectroscopy of nuclei from exotic beams will be performed after in-flight isotope separation. The exotic beams will be produced by fragmentation of heavy stable primary beams or by fission of a ${ }^{238} \mathrm{U}$ beam on a ${ }^{9} \mathrm{Be}$ or ${ }^{208} \mathrm{~Pb}$ target and then selected by the fragment separator. Other $\gamma$-ray detector arrays are now starting operation at radioactive ion beam facilities such as Spiral (Ganil, France) or Rex-isolde (CERN, Switzerland).

\section{Increasing the sensitivity of germanium-detector arrays by $\gamma$-ray tracking}

Gamma-ray detector arrays of the present generation are built of Compton-suppressed Ge-detectors arranged in tightly packed spherical configurations. High granularity (and large distances from the target position) is needed for an efficient Doppler shift correction.

This is achieved by rejecting the signal from the detector when the surrounding BGO scintillator shield detects $\gamma$-rays that are Compton scattered out of the Ge crystal. It is now the common 
view that in order to make the next major advance, the suppression shields have to be replaced by active Ge detectors. The major problem here is the uncontrollable Compton scattering. A solution is that all $\gamma$-ray interaction paths need to be tracked and characterized-see figure 8 .

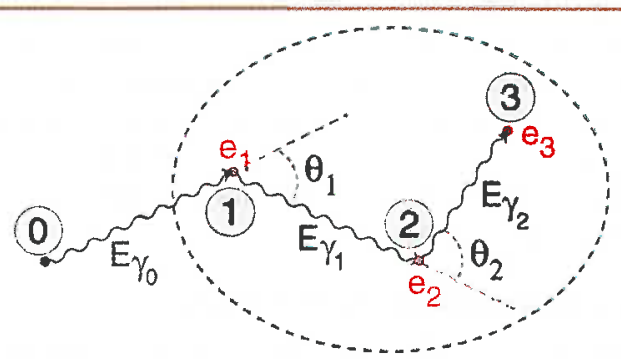

A Fig. 8: A Compton scattering event inside a Ge detector having three interaction points. Tracking of the gamma ray can be achieved through precise measurements of energy deposit and position at each interaction point. Digital electronics and pulse shape analysis are therefore essential. The tracking reconstruction efficiency, and therefore the total efficiency of the system, will be strongly dependent on the position resolution, which can be brought to few millimeters.

The recent advances in crystal segmentation technology and digital signal processing have opened the possibility to operate the detectors in a position-sensitive mode with high counting rates. This enables the design of a compact array solely out of $\mathrm{Ge}$ detectors omitting the BGO shields (Advanced GAmma ray
Tracking Array). It is expected from simulations that an array consisting of a limited number $(\sim 50-100)$ of such detectors can have unprecedented features: an efficiency of up to $\sim 40 \%$ while maintaining a signal over noise-ratio of the order of $60 \%$. Therefore by using the tracking technique one expects to improve the array sensitivity by about two orders of magnitude over the current generation of $\mathrm{Ge}$-arrays. A wide European collaboration is now progressing in this way [15].

\section{References}

[1] Boynton et al., Science 297 (5578):81 R24 and http://grs.lpl.arizona.edu

[2] J.Simpson, Z. Phys A358 (1997)139

[3] NewScientist, 8 June 2002, page 7 and J. Dudek et al. Phys. Rev. Lett. 88 (2002) 252502

[4] G. Benzoni et al., Phys. Lett. B 540 (2002) 199

[5] S. Toermaenen et al., Phys. Lett. B 454 (1999) 8

[6] S.W. Ødegård et al., Phys. Rev. Lett. 86 (2001) 5866

[7] D.R. Jensen et al, Phys. Rev. Lett. 89 (2002) 142503

[8] W.von Oertzen, Eur. Phys. J.A11 (2001) 403

[9] G. Baldsiefen et al., Nucl. Phys. A 574 (1994) 521

[10] R.M. Lieder et al., Eur. Phys. J.A13 (2002) 297

[11] S. Frauendorf, Nucl. Phys. A 557 (1993) 259c

[12] S. Lenzi et al., Phys. Rev. Lett. 87 (2001) 12250

[13] E. Farnea et al., Phys. Lett. B (2002)

[14] C. Fahlander et al., Phys. Rev. C 63 (2001) 021307(R)

[15] R. Lieder et al., Nucl. Phys. A682(2001)279c

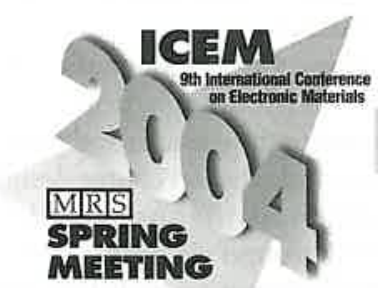

APRIL 12-16

SAN FRANCISCO, CA USA

\begin{tabular}{c} 
ABSTRACT DEADLINES: \\
October 20, 2003 \\
for abstracts sent \\
via fax or mail \\
November 3,2003 \\
for abstracts sent \\
via the MRS Web site \\
\hline in faimess to all potential \\
authors, late abstracts will \\
nol be accepled.
\end{tabular}

For additional meeting information, visit the MRS Web site at

wur.mrs.org/meelings/ or contact:

\section{M]RS}

Member Services

Materials Research Socieby 506 Keystone Drive

Warrendale, PA 15086-7573

Tel 724-779-3003

Fax $724-779-8313$

E-mail: info@mrs.org

\section{MPS SPRING MIFFING}

\section{CALL FOR PAPERS}

wrw.mrs.org/meetings/spring2004/

\begin{tabular}{|c|c|c|}
\hline \multicolumn{3}{|l|}{ SGHEDULED SYMPOSIA } \\
\hline $\begin{array}{l}\text { ELECTRONICS, SPINTRONICS, AND PHDTONICS } \\
\text { A: Amorphous and Nanocrystalline Silicon Science } \\
\text { and Technology-2004 } \\
\text { B: High-Mobility Group-IV Materials and Devices } \\
\text { C: Silicon Front-End Junction Formation- } \\
\text { Physlcs and Technology } \\
\text { D: High-k Insulators and Ferroelectrics for Advanced } \\
\quad \text { Microelectronic Devices } \\
\text { E: Integration Challenges in Next-Generation } \\
\text { Oxide-Based Nanoetectronics } \\
\text { F: Materials, Technology, and Reliability for } \\
\text { Advanced Interconnects and Low-k Dielectrics } \\
\text { G: Semiconductor Spintronics } \\
\text { H: Hydrogen in Serniconductors } \\
\text { J: Flexible Electronics-Materials and } \\
\text { J: Device Technology } \\
\text { Silicon Carblde-Materials, Processing, } \\
\text { and Devices } \\
\text { L: Advances In Chemical Mechanical Polishing } \\
\text { New Materials for Micropholonics }\end{array}$ & $\begin{array}{l}\text { NANO-AND MICAOSTRUCTURED MATERIALS } \\
\text { M: Nanoparticles and Nanowire Building Blocks } \\
\text { Synthesis, Processing, Characterization, } \\
\text { and Theory } \\
\text { N: Interfacial Engineering for Optimized Properties III } \\
\text { O: Advanced Microsystems-Integration with } \\
\text { Nanotechnology and Biomaterials } \\
\text { P: Nanoscale Materials and Modeling- } \\
\text { Relations Among Processing, Microstructure, } \\
\text { and Mechanical Properties } \\
\text { Q: Nucleation Phenomena-Mechanlsms, } \\
\text { Dynarnics, and Structure } \\
\text { R: Three-Dimensional Nanoenglneered Assemblies II } \\
\text { S: Nanostructured Materials in Alternalive } \\
\text { Energy Devices } \\
\text { MOLECULAR, BIOLOGICAL, AND } \\
\text { HYBHID MATERIALS } \\
\text { T: Molecular Electronics } \\
\text { U: Printing of Materials in Photonics, Electronics } \\
\text { and Bioinlormatics }\end{array}$ & $\begin{array}{l}\text { V: Proteins as Materials } \\
\text { W: Biological and Bio-Inspired Materials and Devices } \\
\text { Y: Materials, Mechanisms, and Systems for } \\
\text { Chemical and Biological Detection and } \\
\text { Remediation } \\
\text { Z: Hybrid Biological-Inorganic Interlaces } \\
\text { AA: Applicalions of Novel Luminescent Probes } \\
\text { in Life Sciences } \\
\text { GENERAL } \\
\text { X: Frontiers of Materials Research } \\
\text { BB: Educaling Tomorrow's Materials Scientists } \\
\text { and Engineers } \\
\text { CC: Scientific Basis for Nuclear Waste } \\
\text { Management XOVIII }\end{array}$ \\
\hline MEETING ACTWUILS & & \\
\hline $\begin{array}{l}\text { SYMPOSIUM TUTORIAL PROGRAM } \\
\text { Available only to meeting registrants, the } \\
\text { symposium tutorials will concentrate on new, } \\
\text { rapidly breaking areas of research. } \\
\text { EXHIBIT } \\
\text { A major exhibit encompassing the full spectrum } \\
\text { of equipment, instrumentation, products, } \\
\text { software, publications, and services is } \\
\text { scheduled for April } 13-15 \text { in Moscone West, } \\
\text { convenient to the technical session rooms. }\end{array}$ & $\begin{array}{l}\text { PUBLICATIONS DESK } \\
\text { A full display of over } 800 \text { books will be available } \\
\text { at the MRS Publications Desk. Symposium } \\
\text { Proceedings from both the } 2003 \text { MRS Spring } \\
\text { and Fall Meetings will be featured. }\end{array}$ & $\begin{array}{l}\text { CAREER CENTER } \\
\text { A Career Center for MRS members and meeting } \\
\text { attendees will be offered in Moscone West } \\
\text { during the } 2004 \text { MRS Spring Meeting. }\end{array}$ \\
\hline
\end{tabular}

